\title{
Numerical Solution of Two-Point Boundary Value Problems by Interpolating Subdivision Schemes
}

\author{
Ghulam Mustafa and Syeda Tehmina Ejaz \\ Department of Mathematics, The Islamia University of Bahawalpur, Punjab 63100, Pakistan \\ Correspondence should be addressed to Ghulam Mustafa; ghulam.mustafa@iub.edu.pk
}

Received 23 January 2014; Revised 9 June 2014; Accepted 22 June 2014; Published 17 July 2014

Academic Editor: Chun-Gang Zhu

Copyright ( 2014 G. Mustafa and S. T. Ejaz. This is an open access article distributed under the Creative Commons Attribution License, which permits unrestricted use, distribution, and reproduction in any medium, provided the original work is properly cited.

\begin{abstract}
A numerical interpolating algorithm of collocation is formulated, based on 8-point binary interpolating subdivision schemes for the generation of curves, to solve the two-point third order boundary value problems. It is observed that the algorithm produces smooth continuous solutions of the problems. Numerical examples are given to illustrate the algorithm and its convergence. Moreover, the approximation properties of the collocation algorithm have also been discussed.
\end{abstract}

\section{Introduction}

Subdivision plays an important role in computer aided geometric design. Particularly, subdivision schemes for curve design consist of repeated refinement of control polygons. In particular, the linear schemes are well studied with rules for defining the control points at the finer level as finite linear combinations of the control points in the coarser level.

The concept of subdivision has been initiated by de Rham [1]. Later on, in [2], Dyn et al. studied a family of schemes with mask of size four, indexed by a tension parameter. In [3], Dubuc and Deslauriers studied a family of schemes indexed by the size of the mask $2 m$ and the arity (or base) $b$; that is, at each subdivision step, the scheme $(m, b)$ uses masks of $2 m$ points to compute $b$ new points corresponding to each old edge. Mustafa and Rehman [4] unified all existing even-point interpolating and approximating schemes by offering general formula for the mask of $(2 m+4)$-point even-ary subdivision scheme. Aslam et al. [5] presented an explicit formula which unified the mask of $(2 m-1)$-point interpolating as well as approximating schemes. Mustafa et al. [6] presented an explicit formula for the mask of odd-points $b$-ary (for any odd $m \geq 3$ ) interpolating subdivision schemes. Higher arity schemes have been introduced in $[7,8]$. Mustafa and Ivrissimitzis [9] have showed that when subdivision is used as a data modelling tool large support, or large arity schemes, which generally produce smoother curves, it may outperform simpler schemes.

An interpolating subdivision scheme approach to the construction of approximate solutions of two-point second order boundary value problems was first time introduced in [10]. In this approach a method of collocation was formulated for linear two-point second order boundary value problems. It is proved that the algorithms produce smooth continuous solutions provided the algorithms are chosen appropriately. Later on, in [11], they reformulated the collocation method by subdivision scheme in order to compute numerical solutions for two-point boundary-value problems of differential equations with deviating arguments. They demonstrated that their approach has further development to their previous works for solving various types of two-point second order boundaryvalue problems.

They approximated the derivative boundary conditions by forward difference operator so it is difficult (with no flexibility to improve the solution) to generalize their approach to solve third or higher order boundary value problems. We express derivative boundary conditions at end points by using interpolatory subdivision algorithm; therefore it is easy to generalize our approach to deal with higher order boundary value problems.

In this paper, we have reformulated the collocation algorithm by using 8 -point interpolating subdivision scheme 
to compute the approximate solution of two-point third order boundary value problems. Particularly, collocation algorithm with septic precision treatments at the endpoints has the power of approximation $\mathrm{O}\left(h^{2}\right)$. Our reformulated collocation algorithm treats the following type of two-point third order boundary value problem:

$$
\begin{aligned}
y^{\prime \prime \prime}(x) & =a(x) y(x)+b(x), & & 0 \leq x \leq 1, \\
y(0) & =y_{r}, & y^{\prime}(0)=0, & y(1)=y_{l},
\end{aligned}
$$

where $a(x)$ and $b(x)$ are continuous and $a(x) \geq 0$ on $[0,1]$.

The outline of the paper is as follows. In Section 2, we rewrite general form of interpolating subdivision scheme for curve design [12] and some related results. The 8-point binary interpolating scheme and derivatives of its basis function have also been discussed in this section. In Section 3, a numerical interpolating algorithm of collocation to solve (1) is formulated and its boundary treatments are discussed. In Section 4, approximation properties of this algorithm are given. In Section 5, numerical examples are presented. Section 6 is devoted for conclusion and the possible future research directions.

\section{Interpolating Schemes for Curve Design}

A general compact form of symmetric univariate binary interpolating subdivision scheme [12] which maps polygon $p^{k}=\left\{p_{i}^{k}\right\}_{i \in \mathbb{Z}}$ to a refined polygon $p^{k+1}=\left\{p_{i}^{k+1}\right\}_{i \in \mathbb{Z}}$ is defined by

$$
\begin{gathered}
p_{2 i}^{k+1}=p_{i}^{k} \\
p_{2 i+1}^{k+1}=\sum_{j=0}^{n} L_{n, j}\left(p_{i-j}^{k}+p_{i+j+1}^{k}\right),
\end{gathered}
$$

where $n$ is called the degree of the scheme and the constants are given by

$$
\begin{array}{r}
L_{n, j}=\frac{((2 n+1) ! !)^{2}}{2\left(4^{n}\right)(2 n+1) !} \frac{(-1)^{j}}{(2 j+1)}\left(\begin{array}{c}
2 n+1 \\
n-j
\end{array}\right), \\
j=0,1,2, \ldots, n,
\end{array}
$$

where $\left(\begin{array}{c}2 n+1 \\ n-j\end{array}\right)$ denotes the binomial coefficient.

The boundary treatments are necessary to produce smooth curve segments by scheme (2). Normally higher order approximation formulae should be used near the ends of the segments and thus Lagrange formulae of order $2 n+1$ are recommended.

Remark 1. Let $\phi(x)$ be the limit curve generated from the cardinal data $\left\{p_{i}=\left(i, \delta_{0}\right)^{T}\right\}$; that is, $\phi(x)$ is the fundamental solution of the subdivision scheme (2); then

$$
\phi(i)= \begin{cases}1, & i=0, \\ 0, & i \neq 0 .\end{cases}
$$

Furthermore, $\phi(x)$ satisfies the following two-scale equation:

$$
\begin{aligned}
\phi(x) & =\phi_{n}(x) \\
& =\phi(2 x)+\sum_{j=-n}^{n} L_{n,|j|} \phi(2 x-2 j+1), \quad x \in \mathbb{R} .
\end{aligned}
$$

Lemma 2 (see $[12,13]$ ). The support of the fundamental solution $\phi_{n}(x)$ to scheme (2) is finite. Explicitly, supp $\phi_{n}(x)=$ $(-2 n-1,2 n+1)$.

Lemma 3 (see [10]). Given a square matrix $A$ of order $n$, let the normalized left and right (generalized) eigenvectors of $A$ be denoted by $\left\{\eta_{i}, \xi_{i}\right\}$. Then for any vector $f \in \mathbb{R}^{n}$, there exists following Fourier expansion:

$$
f=\sum_{i=1}^{n}\left(f^{T} \eta_{i}\right) \xi_{i} .
$$

Lemma 4 (see $[10])$. Suppose $F(t), t \in \mathbb{R}$ is a regular and $C^{2 n+2}$ curve in $\mathbb{R}^{m}, m \geq 2$. Let $P(t), t \in \mathbb{R}$ be the limit curve generated by (2) from the initial data $P_{i}=F(i h), i \in \mathbb{Z}, 0<h<1$. Then, on any finite interval $[a, b]$, the following estimates hold:

$$
\begin{gathered}
\|F(h t)-p(t)\|_{\infty} \leq \frac{M_{2 n+2}(F)}{(2 n+2) !} h^{2 n+2}=O\left(h^{2 n+2}\right), \\
\left\|h^{j} F^{j}(h t)-p^{j}(t)\right\|_{\infty}=O\left(h^{2 n+2-j}\right), \\
j=0,1,2, \ldots, \frac{n+2}{2},
\end{gathered}
$$

where the number $M_{2 n+2}(F)$ depends only on the derivatives of $F(t)$ and $n$.

2.1. 8-Point Interpolating Scheme. For $n=3$ by (2) and (3), we have the following 8-point binary interpolating subdivision scheme for curve design:

$$
\begin{gathered}
p_{2 i}^{k+1}=p_{i}^{k} \\
p_{2 i+1}^{k+1}=\frac{1225}{2048}\left(p_{i}^{k}-p_{i+1}^{k}\right)-\frac{245}{2048}\left(p_{i-1}^{k}-p_{i+2}^{k}\right) \\
+\frac{49}{2048}\left(p_{i-2}^{k}-p_{i+3}^{k}\right)-\frac{5}{2048}\left(p_{i-3}^{k}-p_{i+4}^{k}\right) .
\end{gathered}
$$

This scheme is $C^{3}$-continuous in [14] and reproduces polynomial curve of degree seven in [15]. The local subdivision matrix of (8) is denoted and defined by 


$$
S=\left(\begin{array}{ccccccccccccc}
0 & 0 & 0 & 1 & 0 & 0 & 0 & 0 & 0 & 0 & 0 & 0 & 0 \\
L_{3,3} & L_{3,2} & L_{3,1} & L_{3,0} & L_{3,0} & L_{3,1} & L_{3,2} & L_{3,3} & 0 & 0 & 0 & 0 & 0 \\
0 & 0 & 0 & 0 & 1 & 0 & 0 & 0 & 0 & 0 & 0 & 0 & 0 \\
0 & L_{3,3} & L_{3,2} & L_{3,1} & L_{3,0} & L_{3,0} & L_{3,1} & L_{3,2} & L_{3,3} & 0 & 0 & 0 & 0 \\
0 & 0 & 0 & 0 & 0 & 1 & 0 & 0 & 0 & 0 & 0 & 0 & 0 \\
0 & 0 & L_{3,3} & L_{3,2} & L_{3,1} & L_{3,0} & L_{3,0} & L_{3,1} & L_{3,2} & L_{3,3} & 0 & 0 & 0 \\
0 & 0 & 0 & 0 & 0 & 0 & 1 & 0 & 0 & 0 & 0 & 0 & 0 \\
0 & 0 & 0 & L_{3,3} & L_{3,2} & L_{3,1} & L_{3,0} & L_{3,0} & L_{3,1} & L_{3,2} & L_{3,3} & 0 & 0 \\
0 & 0 & 0 & 0 & 0 & 0 & 0 & 1 & 0 & 0 & 0 & 0 & 0 \\
0 & 0 & 0 & 0 & L_{3,3} & L_{3,2} & L_{3,1} & L_{3,0} & L_{3,0} & L_{3,1} & L_{3,2} & L_{3,3} & 0 \\
0 & 0 & 0 & 0 & 0 & 0 & 0 & 0 & 1 & 0 & 0 & 0 & 0 \\
0 & 0 & 0 & 0 & 0 & L_{3,3} & L_{3,2} & L_{3,1} & L_{3,0} & L_{3,0} & L_{3,1} & L_{3,2} & L_{3,3} \\
0 & 0 & 0 & 0 & 0 & 0 & 0 & 0 & 0 & 1 & 0 & 0 & 0
\end{array}\right),
$$

where $L_{3,0}=1225 / 2048, L_{3,1}=-245 / 2048, L_{3,2}=49 / 2048$, and $L_{3,3}=-5 / 2048$. The some of its eigenvalues is

$$
\lambda=1, \frac{1}{2}, \frac{1}{4}, \frac{1}{8}, \frac{1}{16}, \frac{1}{32}, \frac{1}{64}, \frac{1}{128} .
$$

For an eigenvalue $\lambda$, the eigenvectors $\xi$ and $\eta$ that satisfy $S \xi=$ $\lambda \xi$ and $\eta S^{T}=\eta \lambda$ are called right and left eigenvectors of the matrix $S$, respectively. Some of the normalized left and right eigenvectors corresponding to eigenvalues are

$$
\begin{aligned}
& \xi_{0}=(1,1,1,1,1,1,1,1,1,1,1,1,1)^{T}, \\
& \eta_{0}=(0,0,0,0,0,0,1,0,0,0,0,0,0)^{T}, \\
& \xi_{1}=(-6,-5,-4,-3,-2,-1,0,1,2,3,4,5,6)^{T}, \\
& \eta_{1}=\left(\frac{1}{5946360}\right)(-5,1024,13225,-199680,1141695, \\
& -4715520,0,4715520,-1141695, \\
& 199680,-13225,-1024,5)^{T} \text {, } \\
& \xi_{2}=(36,25,16,9,4,1,0,1,4,9,16,25,36)^{T}, \\
& \eta_{2}=\left(\frac{1}{34546860}\right)(275,-28160,-182613,2607616 \text {, } \\
& \text { - 12053651, 45634048, -71955030, } \\
& \text { 45634048, -12053651, 2607616, } \\
& -182613,-28160,275)^{T}, \\
& \xi_{3}=(-216,-125,-64,-27,-8,-1,0,1,8,27,64,125,216)^{T}, \\
& \eta_{3}=\left(\frac{1}{15039360}\right)(225,-11520,10952,476928,
\end{aligned}
$$$$
\text { - 3047987, 4677632, 0, -4677632, }
$$$$
3047987,-476928,-10952 \text {, }
$$$$
11520,-225)^{T} \text {. }
$$

Since $\xi_{i}^{T} \eta_{j}=1$ for $i=j$ and 0 otherwise then by using Lemmas 2 and 3 , we get the following result.

Lemma 5. The fundamental solution (Cardinal basis) $\phi(x)$ is thrice continuously differentiable and supported on $(-7,7)$ and its derivatives at integers are given by

$$
\begin{aligned}
\phi^{\prime}(i) & =2 \operatorname{sign}(i) e_{|i|}^{T} \eta_{1}, \\
\phi^{\prime \prime}(i) & =2^{2} e_{|i|}^{T} \eta_{2}, \\
\phi^{\prime \prime \prime}(i) & =2^{3} \operatorname{sign}(i) e_{|i|}^{T} \eta_{3}, \\
& -6 \leq i \leq 6,
\end{aligned}
$$

where

$$
\begin{aligned}
& e_{0}=(0,0,0,0,0,0,1,0,0,0,0,0,0)^{T}, \\
& e_{1}=(0,0,0,0,0,1,0,0,0,0,0,0,0)^{T}, \\
& e_{2}=(0,0,0,0,1,0,0,0,0,0,0,0,0)^{T}, \\
& e_{3}=(0,0,0,1,0,0,0,0,0,0,0,0,0)^{T}, \\
& e_{4}=(0,0,1,0,0,0,0,0,0,0,0,0,0)^{T}, \\
& e_{5}=(0,1,0,0,0,0,0,0,0,0,0,0,0)^{T}, \\
& e_{6}=(1,0,0,0,0,0,0,0,0,0,0,0,0)^{T}, \\
& \phi^{\prime}(0)=0, \quad \phi^{\prime}( \pm 1)=\mp \frac{78592}{49553}, \\
& \phi^{\prime}( \pm 2)= \pm \frac{76113}{198212}, \quad \phi^{\prime}( \pm 3)=\mp \frac{3328}{49553}, \\
& \phi^{\prime}( \pm 4)= \pm \frac{2645}{594636}, \quad \phi^{\prime}( \pm 5)= \pm \frac{256}{743295}, \\
& \phi^{\prime}( \pm 6)=\frac{1}{594636},
\end{aligned}
$$




$$
\begin{gathered}
\phi^{\prime \prime}(0)=-\frac{342643}{41124}, \quad \phi^{\prime \prime}( \pm 1)=\frac{5704256}{1079505}, \\
\phi^{\prime \prime}( \pm 2)=-\frac{12053651}{8636040}, \quad \phi^{\prime \prime}( \pm 3)=\frac{325952}{1079505}, \\
\phi^{\prime \prime}( \pm 4)=-\frac{60871}{2878680}, \quad \phi^{\prime \prime}( \pm 5)=-\frac{704}{215901}, \\
\phi^{\prime \prime \prime}( \pm 6)=\frac{55}{1727208}, \\
\phi^{\prime \prime \prime}( \pm 2)= \pm \frac{3047987}{1879920}, \quad \phi^{\prime \prime \prime}( \pm 1)=\mp \frac{292352}{117495}, \\
\phi^{\prime \prime \prime}( \pm 4)=\mp \frac{1369}{234990}, \quad \phi^{\prime \prime \prime}( \pm 5)= \pm \frac{3312}{13055}, \\
\phi^{\prime \prime \prime}( \pm 6)=\mp \frac{5}{41776} .
\end{gathered}
$$

The graphical representations of the basis limit function defined on cardinal data and its derivatives up to order three for $n=3$ are shown in Figure 1. Figure 1(a) represents the basis limit function defined in (4). Graphical representations of first, second and third derivatives of basis limit functions obtained from (5) for $n=3$ are shown in Figures 1(b), 1(c) and $1(\mathrm{~d})$ at $i=0,1,-1$, respectively. The numeric values of first, second and third derivative are given in (13).

\section{Numerical Interpolating Collocation Algorithm}

In this section, first we formulate a numerical interpolating collocation algorithm for linear third order two-point boundary value problems. Then we settle down the boundary conditions to get unique solution.

3.1. The Collocation Algorithm. Let $N$ be a positive integer $(N \geq 6), h=1 / N$, and $x_{i}=i / N=i h, i=0,1,2, \ldots, N$, and set $a_{i}=a\left(x_{i}\right), b_{i}=b\left(x_{i}\right)$. Let

$$
Z(x)=\sum_{i=-6}^{N+6} z_{i} \phi\left(\frac{x-x_{i}}{h}\right), \quad 0 \leq x \leq 1
$$

be the approximate solution to (1), where $\left\{z_{i}\right\}$ are the unknown to be determined by (1). The collocation algorithm, together with the boundary conditions to be discussed, is given by setting

$$
Z^{\prime \prime \prime}\left(x_{j}\right)=a\left(x_{j}\right) Z\left(x_{j}\right)+b\left(x_{j}\right), \quad j=0,1,2, \ldots, N,
$$

where

$$
\begin{aligned}
Z^{\prime}\left(x_{j}\right) & =\frac{1}{h} \sum_{i=-6}^{N+6} z_{i} \phi^{\prime}\left(\frac{x_{j}-x_{i}}{h}\right), \\
Z^{\prime \prime}\left(x_{j}\right) & =\frac{1}{h^{2}} \sum_{i=-6}^{N+6} z_{i} \phi^{\prime \prime}\left(\frac{x_{j}-x_{i}}{h}\right), \\
Z^{\prime \prime \prime}\left(x_{j}\right) & =\frac{1}{h^{3}} \sum_{i=-6}^{N+6} z_{i} \phi^{\prime \prime \prime}\left(\frac{x_{j}-x_{i}}{h}\right) .
\end{aligned}
$$

Using (14) and (16) in (15), we get following $N+1$ system of equations:

$$
\begin{array}{r}
\sum_{i=-6}^{N+6} z_{i} \phi^{\prime \prime \prime}\left(\frac{x_{j}-x_{i}}{h}\right)-h^{3} a_{j} \sum_{i=-6}^{N+6} z_{i} \phi\left(\frac{x_{j}-x_{i}}{h}\right)=h^{3} b_{j}, \\
j=0,1,2, \ldots, N .
\end{array}
$$

Now we simplify the above system in the following theorems.

Theorem 6. For $j=0$ by (17), one gets

$$
\begin{aligned}
z_{-6} \phi_{6}^{\prime \prime \prime} & +z_{-5} \phi_{5}^{\prime \prime \prime}+z_{-4} \phi_{4}^{\prime \prime \prime}+z_{-3} \phi_{3}^{\prime \prime \prime}+z_{-2} \phi_{2}^{\prime \prime \prime} \\
& +z_{-1} \phi_{1}^{\prime \prime \prime}+z_{0} q_{0}+z_{1} \phi_{-1}^{\prime \prime \prime}+z_{2} \phi_{-2}^{\prime \prime \prime}+z_{3} \phi_{-3}^{\prime \prime \prime} \\
& +z_{4} \phi_{-4}^{\prime \prime \prime}+z_{5} \phi_{-5}^{\prime \prime \prime}+z_{6} \phi_{-6}^{\prime \prime \prime}=h^{3} b_{0},
\end{aligned}
$$

where $\phi_{j}^{\prime \prime \prime}=\phi^{\prime \prime \prime}(j)$ and $q_{0}=\phi_{0}^{\prime \prime \prime}-a_{0} h^{3}$.

Proof. Substituting $j=0$ in (17), we get

$$
\begin{aligned}
& \left\{z_{-6} \phi^{\prime \prime \prime}\left(\frac{x_{0}-x_{-6}}{h}\right)+z_{-5} \phi^{\prime \prime \prime}\left(\frac{x_{0}-x_{-5}}{h}\right)+\cdots\right. \\
& \left.+z_{N+5} \phi^{\prime \prime \prime}\left(\frac{x_{0}-x_{N+5}}{h}\right)+z_{N+6} \phi^{\prime \prime \prime}\left(\frac{x_{0}-x_{N+6}}{h}\right)\right\} \\
& -a_{0} h^{3}\left\{z_{-6} \phi\left(\frac{x_{0}-x_{-6}}{h}\right)+z_{-5} \phi\left(\frac{x_{0}-x_{-5}}{h}\right)+\cdots\right. \\
& \left.+z_{N+5} \phi\left(\frac{x_{0}-x_{N+5}}{h}\right)+z_{N+6} \phi\left(\frac{x_{0}-x_{N+6}}{h}\right)\right\} \\
& =h^{3} b_{0} .
\end{aligned}
$$

For $x_{j}=j h, j=0,1,2 \ldots, N$, this implies

$$
\begin{aligned}
& z_{-6} \phi^{\prime \prime \prime}(6)+z_{-5} \phi^{\prime \prime \prime}(5)+\cdots+z_{N+5} \phi^{\prime \prime \prime}(-N-5) \\
& +z_{N+6} \phi^{\prime \prime \prime}(-N-6) \\
& -a_{0} h^{3}\left\{z_{-6} \phi(6)+z_{-5} \phi(5)+\cdots\right. \\
& \left.\quad+z_{N+5} \phi(-N-5)+z_{N+6} \phi(-N-6)\right\}=h^{3} b_{0} .
\end{aligned}
$$




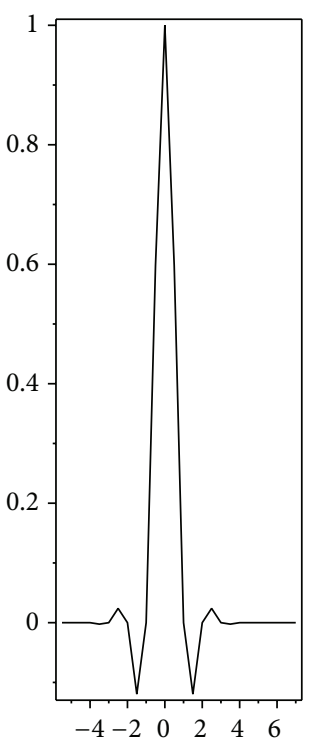

(a)

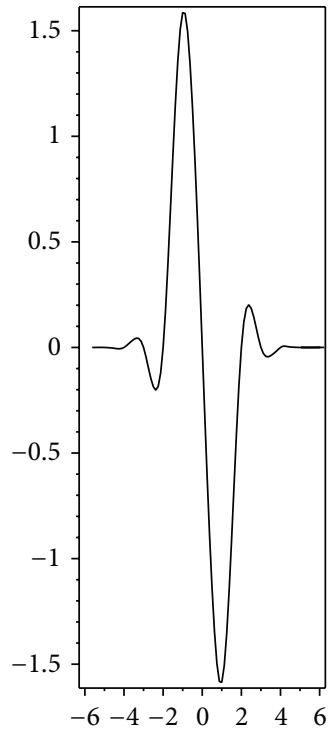

(b)

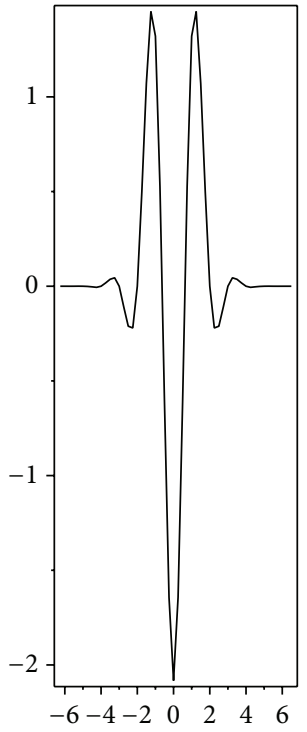

(c)

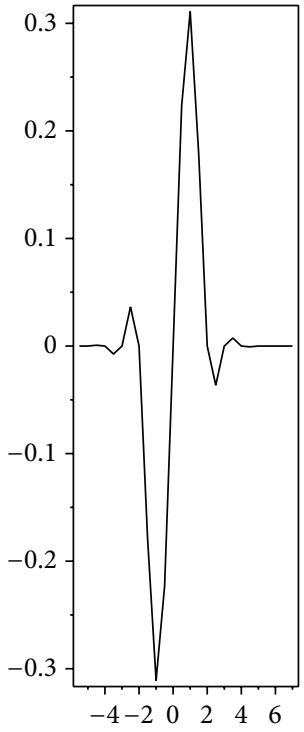

(d)

Figure 1: Interpolatory basis function $\phi_{3}(i)$ is shown in (a), first derivative of $\phi_{3}(i)$ i-e. $\phi_{3}^{\prime}(i)$ shown in (b), second derivative of $\phi_{3}(i)$ i-e. $\phi_{3}^{\prime \prime}(i)$ shown in (c), and third derivative of $\phi_{3}(i)$ i-e. $\phi_{3}^{\prime \prime \prime}(i)$ shown in $(d)$, respectively.

Since the support of basis function $\phi(x)$ is $(-7,7), \phi^{\prime}(x)$, $\phi^{\prime \prime}(x)$, and $\phi^{\prime \prime \prime}(x)$ are zero outside the interval $(-7,7)$; also by (4) and (13), we get

$$
\begin{aligned}
z_{-6} \phi^{\prime \prime \prime} & (6)+z_{-5} \phi^{\prime \prime \prime}(5)+z_{-4} \phi^{\prime \prime \prime}(4)+z_{-3} \phi^{\prime \prime \prime} \\
& +z_{-2} \phi^{\prime \prime \prime}(2)+z_{-1} \phi^{\prime \prime \prime}(1)+z_{0} \phi^{\prime \prime \prime}(0) \\
& +z_{1} \phi^{\prime \prime \prime}(-1)+z_{2} \phi^{\prime \prime \prime}(-2)+z_{3} \phi^{\prime \prime \prime}(-3) \\
& +z_{4} \phi^{\prime \prime \prime}(-4)+z_{5} \phi^{\prime \prime \prime}(-5)+z_{6} \phi^{\prime \prime \prime}(-6) \\
& -a_{0} h^{3} z_{0} \phi(0)=h^{3} b_{0} .
\end{aligned}
$$

If $\phi_{j}^{\prime \prime \prime}=\phi^{\prime \prime \prime}(j)$, then

$$
\begin{aligned}
z_{-6} \phi_{6}^{\prime \prime \prime} & +z_{-5} \phi_{5}^{\prime \prime \prime}+z_{-4} \phi_{4}^{\prime \prime \prime}+z_{-3} \phi_{3}^{\prime \prime \prime}+z_{-2} \phi_{2}^{\prime \prime \prime} \\
& +z_{-1} \phi_{1}^{\prime \prime \prime}+z_{0}\left(\phi_{0}^{\prime \prime \prime}-a_{0} h^{3}\right)+z_{1} \phi_{-1}^{\prime \prime \prime}+z_{2} \phi_{-2}^{\prime \prime \prime} \\
& +z_{3} \phi_{-3}^{\prime \prime \prime}+z_{4} \phi_{-4}^{\prime \prime \prime}+z_{5} \phi_{-5}^{\prime \prime \prime}+z_{6} \phi_{-6}^{\prime \prime \prime}=h^{3} b_{0} .
\end{aligned}
$$

For $q_{0}=\phi_{0}^{\prime \prime \prime}-a_{0} h^{3}$, we get (18). This completes the proof.

Theorem 7. For $j=1,2,3, \ldots, N$ the system (17) is equivalent to

$$
\begin{aligned}
& z_{-6} \phi_{j+6}^{\prime \prime \prime}+z_{-5} \phi_{j+5}^{\prime \prime \prime}+\cdots+z_{0} \phi_{j}^{\prime \prime \prime} \\
& \quad+z_{1}\left(\phi_{j-1}^{\prime \prime \prime}-a_{j} h^{3} \phi_{j-1}\right)+z_{2}\left(\phi_{j-2}^{\prime \prime \prime}-a_{j} h^{3} \phi_{j-2}\right)+\cdots \\
& \quad+z_{N-1}\left(\phi_{j-N+1}^{\prime \prime \prime}-a_{j} h^{3} \phi_{j-N+1}\right)+z_{N}\left(\phi_{j-N}^{\prime \prime \prime}-a_{j} h^{3} \phi_{j-N}\right) \\
& \quad+z_{N+1} \phi_{j-N-1}^{\prime \prime \prime}+\cdots+z_{N+6} \phi_{j-N-6}^{\prime \prime \prime}=h^{3} b_{j} .
\end{aligned}
$$

Proof. By expanding (17), we get

$$
\begin{aligned}
& z_{-6} \phi^{\prime \prime \prime}\left(\frac{x_{j}-x_{-6}}{h}\right)+z_{-5} \phi^{\prime \prime \prime}\left(\frac{x_{j}-x_{-5}}{h}\right)+\cdots \\
& +z_{N+5} \phi^{\prime \prime \prime}\left(\frac{x_{j}-x_{N+5}}{h}\right)+z_{N+6} \phi^{\prime \prime \prime}\left(\frac{x_{j}-x_{N+6}}{h}\right) \\
& -a_{j} h^{3}\left\{z_{-6} \phi\left(\frac{x_{j}-x_{-6}}{h}\right)+z_{-5} \phi\left(\frac{x_{j}-x_{-5}}{h}\right)+\cdots\right. \\
& \left.+z_{N+5} \phi\left(\frac{x_{j}-x_{N+5}}{h}\right)+z_{N+6} \phi\left(\frac{x_{j}-x_{N+6}}{h}\right)\right\} \\
& =h^{3} b_{j} .
\end{aligned}
$$

For $x_{j}=j h, j=1,2, \ldots, N$, we get

$$
\begin{aligned}
& z_{-6} \phi^{\prime \prime \prime}(j+6)+z_{-5} \phi^{\prime \prime \prime}(j+5)+\cdots \\
& +z_{N+5} \phi^{\prime \prime \prime}(j-N-5)+z_{N+6} \phi^{\prime \prime \prime}(j-N-6) \\
& -a_{j} h^{3}\left\{z_{-6} \phi(j+6)+z_{-5} \phi(j+5)+\cdots\right. \\
& \left.\quad+z_{N+5} \phi(j-N-5)+z_{N+6} \phi(j-N-6)\right\} \\
& =h^{3} b_{j} .
\end{aligned}
$$


If $\phi_{j}^{\prime \prime \prime}=\phi^{\prime \prime \prime}(j)$, for $j=1,2, \ldots, N$, then

$$
\begin{gathered}
z_{-6}\left(\phi_{j+6}^{\prime \prime \prime}-a_{j} h^{3} \phi_{j+6}\right)+z_{-5}\left(\phi_{j+5}^{\prime \prime \prime}-a_{j} h^{3} \phi_{j+5}\right) \\
+\cdots+z_{N+5}\left(\phi_{j-N-5}^{\prime \prime \prime}-a_{j} h^{3} \phi_{j-N-5}\right) \\
+z_{N+6}\left(\phi_{j-N-6}^{\prime \prime \prime}-a_{j} h^{3} \phi_{j-N-6}\right)=h^{3} b_{j} .
\end{gathered}
$$

Since $\phi^{\prime}(x), \phi^{\prime \prime}(x)$, and $\phi^{\prime \prime \prime}(x)$ are zero outside the interval $(-7,7)$ then, by $(4)$ and $(13)$, we get $(23)$.

From (18) and (23), we get following undetermined system of $(N+1)$ equations with $(N+13)$ unknowns $\left\{z_{i}\right\}$ :

$$
A Z=D
$$

where the matrices $A, Z$, and $D$ of orders $(N+1) \times(N+13)$, $N+13$, and $N+1$, respectively, are given by

$$
A=\left(\begin{array}{ccccccccccccccccc}
\phi_{6}^{\prime \prime \prime} & \phi_{5}^{\prime \prime \prime} & \phi_{4}^{\prime \prime \prime} & \phi_{3}^{\prime \prime \prime} & \phi_{2}^{\prime \prime \prime} & \phi_{1}^{\prime \prime \prime} & q_{0} & \phi_{-1}^{\prime \prime \prime} & \phi_{-2}^{\prime \prime \prime} & \phi_{-3}^{\prime \prime \prime} & \phi_{-4}^{\prime \prime \prime} & \phi_{-5}^{\prime \prime \prime} & \phi_{-6}^{\prime \prime \prime} & \cdots & 0 & 0 & 0 \\
0 & \phi_{6}^{\prime \prime \prime} & \phi_{5}^{\prime \prime \prime} & \phi_{4}^{\prime \prime \prime} & \phi_{3}^{\prime \prime \prime} & \phi_{2}^{\prime \prime \prime} & \phi_{1}^{\prime \prime \prime} & q_{1} & \phi_{-1}^{\prime \prime \prime} & \phi_{-2}^{\prime \prime \prime} & \phi_{-3}^{\prime \prime \prime} & \phi_{-4}^{\prime \prime \prime} & \phi_{-5}^{\prime \prime \prime} & \cdots & 0 & 0 & 0 \\
0 & 0 & \phi_{6}^{\prime \prime \prime} & \phi_{5}^{\prime \prime \prime} & \phi_{4}^{\prime \prime \prime} & \phi_{3}^{\prime \prime \prime} & \phi_{2}^{\prime \prime \prime} & \phi_{1}^{\prime \prime \prime} & q_{2} & \phi_{-1}^{\prime \prime \prime} & \phi_{-2}^{\prime \prime \prime} & \phi_{-3}^{\prime \prime \prime} & \phi_{-4}^{\prime \prime \prime} & \cdots & 0 & 0 & 0 \\
0 & 0 & 0 & \phi_{6}^{\prime \prime \prime} & \phi_{5}^{\prime \prime \prime} & \phi_{4}^{\prime \prime \prime} & \phi_{3}^{\prime \prime \prime} & \phi_{2}^{\prime \prime \prime} & \phi_{1}^{\prime \prime \prime} & q_{3} & \phi_{-1}^{\prime \prime \prime} & \phi_{-2}^{\prime \prime \prime} & \phi_{-3}^{\prime \prime \prime} & \cdots & 0 & 0 & 0 \\
\cdots & \cdots & \cdots & \cdots & \cdots & \cdots & \cdots & \cdots & \cdots & \cdots & \cdots & \cdots & \cdots & \cdots & \cdots & \cdots & \cdots \\
0 & 0 & 0 & 0 & 0 & 0 & 0 & 0 & 0 & 0 & 0 & 0 & 0 & \cdots & \phi_{-5}^{\prime \prime \prime} & \phi_{-6}^{\prime \prime \prime} & 0 \\
0 & 0 & 0 & 0 & 0 & 0 & 0 & 0 & 0 & 0 & 0 & 0 & 0 & \cdots & \phi_{-4}^{\prime \prime \prime} & \phi_{-5}^{\prime \prime \prime} & \phi_{-6}^{\prime \prime \prime}
\end{array}\right),
$$

$Z=\left(z_{-6}, z_{-5}, z_{-4}, z_{-3}, z_{-2}, \ldots, z_{N+6}\right)^{T}$, and $D=\left(b_{0} h^{3}, b_{1} h^{3}\right.$, $\left.b_{2} h^{3}, b_{3} h^{3}, \ldots, b_{N} h^{3}\right)^{T}$, where $\phi_{j}^{\prime \prime \prime}=\phi^{\prime \prime \prime}(j)$ and $q_{j}=\phi_{0}^{\prime \prime \prime}-a_{j} h^{3}$.

3.2. Adjustment of Boundary Conditions. The order of the coefficient matrix $(28)$ is $(N+1) \times(N+13)$. In order to get unique solution of the system, we need twelve more conditions. Here we consider only two different cases. In coming section we will show that the approximate solution can be improved by adjusting different boundary conditions.

Case 1. If we assume $z_{0}^{\prime}=0$ (equivalently $z_{0}=y_{r}=$ finite) then two conditions can be achieved by using following given boundary conditions i-e:

$$
z_{0}=y_{r}, \quad z_{0}^{\prime}=0, \quad z_{N}=y_{l}
$$

Still we need ten more conditions to get stable system. Since subdivision scheme reproduces seven degree polynomials, we define boundary conditions of order eight for solution of (27). For simplicity only the left end points are discussed and the values of right end points $z_{N+1}, z_{N+2}, z_{N+3}, z_{N+4}, z_{N+5}$ can be treated similarly.

The values $z_{-5}, z_{-4}, z_{-3}, z_{-2}, z_{-1}$ can be determined by the septic polynomial $q(x)$ interpolating at $\left(x_{i}, z_{i}\right), 0 \leq i \leq 7$. Precisely, we have

$$
z_{-i}=q\left(-x_{i}\right), \quad i=1,2,3,4,5
$$

where

$$
q\left(x_{i}\right)=\sum_{j=1}^{8}\left(\begin{array}{l}
8 \\
j
\end{array}\right)(-1)^{j+1} Z\left(x_{i-j}\right) .
$$

Since by (14) $Z\left(x_{i}\right)=z_{i}$ for $i=1,2,3,4,5$ then, by replacing $x_{i}$ by $-x_{i}$, we have

$$
q\left(-x_{i}\right)=\sum_{j=1}^{8}\left(\begin{array}{l}
8 \\
j
\end{array}\right)(-1)^{j+1} z_{-i+j} .
$$

Hence the following boundary conditions can be employed at the left end:

$$
\sum_{j=0}^{8}\left(\begin{array}{l}
8 \\
j
\end{array}\right)(-1)^{j} z_{-i+j}=0, \quad i=5,4,3,2,1 .
$$

Similarly, for the right end, we can define $z_{i}=q\left(-x_{i}\right), i=$ $N+1, N+2, N+3, N+4, N+5$, and

$$
q\left(x_{i}\right)=\sum_{j=1}^{8}\left(\begin{array}{l}
8 \\
j
\end{array}\right)(-1)^{j+1} z_{i-j} .
$$

So we have the following boundary conditions at the right end:

$$
\begin{gathered}
\sum_{j=0}^{8}\left(\begin{array}{l}
8 \\
j
\end{array}\right)(-1)^{j} z_{i-j}=0, \\
i=N+1, N+2, N+3, N+4, N+5 .
\end{gathered}
$$

Finally, we get the following new system of $(N+13)$ linear equations with $(N+13)$ unknowns $\left\{z_{i}\right\}$, in which $N+1$ equations are obtained from (18) and (23), two equations from boundary conditions (29) and ten from boundary conditions (33) and (35):

$$
B Z=R,
$$

where the coefficients matrix $B=\left(B_{0}^{T}, A^{T}, B_{1}^{T}\right)^{T}, A$ is defined by (28), and $B_{0}$ and $B_{1}$ are formed by (29), (33), and (35) 


$$
B_{0}=\left(\begin{array}{ccccccccccccccccc}
0 & 1 & -8 & 28 & -56 & 70 & -56 & 28 & -8 & 1 & 0 & 0 & 0 & 0 & \cdots & 0 & 0 \\
0 & 0 & 1 & -8 & 28 & -56 & 70 & -56 & 28 & -8 & 1 & 0 & 0 & 0 & \cdots & 0 & 0 \\
0 & 0 & 0 & 1 & -8 & 28 & -56 & 70 & -56 & 28 & -8 & 1 & 0 & 0 & \cdots & 0 & 0 \\
0 & 0 & 0 & 0 & 1 & -8 & 28 & -56 & 70 & -56 & 28 & -8 & 1 & 0 & \cdots & 0 & 0 \\
0 & 0 & 0 & 0 & 0 & 1 & -8 & 28 & -56 & 70 & -56 & 28 & -8 & 1 & \cdots & 0 & 0 \\
0 & 0 & 0 & 0 & 0 & 0 & 1 & 0 & 0 & 0 & 0 & 0 & 0 & 0 & \cdots & 0 & 0
\end{array}\right),
$$

where the first five rows of $B_{0}$ come from (33) and the sixth row comes from (29) at $z_{0}=y_{r}$. Consider

$$
B_{1}=\left(\begin{array}{ccccccccccccccccc}
0 & 0 & \cdots & 0 & 0 & 0 & 0 & 0 & 0 & 0 & 1 & 0 & 0 & 0 & 0 & 0 & 0 \\
0 & 0 & \cdots & 1 & -8 & 28 & -56 & 70 & -56 & 28 & -8 & 1 & 0 & 0 & 0 & 0 & 0 \\
0 & 0 & \cdots & 0 & 1 & -8 & 28 & -56 & 70 & -56 & 28 & -8 & 1 & 0 & 0 & 0 & 0 \\
0 & 0 & \cdots & 0 & 0 & 1 & -8 & 28 & -56 & 70 & -56 & 28 & -8 & 1 & 0 & 0 & 0 \\
0 & 0 & \cdots & 0 & 0 & 0 & 1 & -8 & 28 & -56 & 70 & -56 & 28 & -8 & 1 & 0 & 0 \\
0 & 0 & \cdots & 0 & 0 & 0 & 0 & 1 & -8 & 28 & -56 & 70 & -56 & 28 & -8 & 1 & 0
\end{array}\right),
$$

where first row of $B_{1}$ comes from (29) at $z_{N}=y_{l}$ remaining rows come from (35) and the matrices $Z$ and $R$ are defined as

$$
\begin{gathered}
Z=\left(z_{-6}, z_{-5}, \ldots, z_{N+5}, z_{N+6}\right)^{T}, \\
R=\left(0,0,0,0,0, y_{r}, D^{T}, y_{l}, 0,0,0,0,0\right)^{T} .
\end{gathered}
$$

Case 2. In this case we express the given boundary condition $z_{0}^{\prime}=0$ in the following way.

By using (16) we have

$$
Z^{\prime}\left(x_{j}\right)=\frac{1}{h} \sum_{i=-6}^{N+6} z_{i} \phi^{\prime}\left(\frac{x_{j}-x_{i}}{h}\right) .
$$

As we defined earlier $x_{j}=j h$ if we put $j=0$ we get $x_{0}=0$; the above equation can be written as

$$
Z^{\prime}(0)=\frac{1}{h} \sum_{i=-6}^{N+6} z_{i} \phi^{\prime}(-i)
$$

Since by boundary condition $z_{0}^{\prime}=Z^{\prime}(0)=0$,

$$
\sum_{i=-6}^{N+6} z_{i} \phi^{\prime}(-i)=0
$$

By using (13) we can express above equation as

$$
\begin{aligned}
& \frac{1}{594636} z_{-6}+\frac{256}{743295} z_{-5}+\frac{2645}{594636} z_{-4}-\frac{3328}{49553} z_{-3} \\
& +\frac{76113}{198212} z_{-2}-\frac{78592}{49553} z_{-1}+\frac{78592}{49553} z_{1}-\frac{76113}{198212} z_{2} \\
& +\frac{3328}{49553} z_{3}-\frac{2645}{594636} z_{4}-\frac{256}{743295} z_{5}-\frac{1}{594636} z_{6}=0 .
\end{aligned}
$$

Finally, we get a following new system of $(N+13)$ linear equations with $(N+13)$ unknowns $\left\{z_{i}\right\}$, in which $N+1$ equations are obtained from (18) and (23), two equations from boundary conditions (29) and ten from boundary conditions (33) for $i=5,4,3,2$, (35), and (43):

$$
B Z=R,
$$

where the coefficients matrix $B=\left(\mathbb{B}_{0}^{T}, A^{T}, B_{1}^{T}\right)^{T}, A$ is defined by (28), and $\mathbb{B}_{0}$ and $B_{1}$ formed by (29), (33), (35), and (43) are defined as 


$$
\begin{aligned}
& \mathbb{B}_{0}=\left(\begin{array}{ccccccccc}
0 & 1 & -8 & 28 & -56 & 70 & -56 & 28 & -8 \\
0 & 0 & 1 & -8 & 28 & -56 & 70 & -56 & 28 \\
0 & 0 & 0 & 1 & -8 & 28 & -56 & 70 & -56 \\
0 & 0 & 0 & 0 & 1 & -8 & 28 & -56 & 70 \\
\frac{1}{594636} & \frac{256}{743295} & \frac{2645}{594636} & -\frac{3328}{49553} & \frac{76113}{198212} & -\frac{78592}{49553} & 0 & \frac{78592}{49553} & \frac{76113}{198212} \\
\hline 0 & 0 & 0 & 0 & 0 & 0 & 1 & 0 & 0
\end{array}\right. \\
& \left.\begin{array}{cccccccc}
1 & 0 & 0 & 0 & 0 & \cdots & 0 & 0 \\
-8 & 1 & 0 & 0 & 0 & \cdots & 0 & 0 \\
28 & -8 & 1 & 0 & 0 & \cdots & 0 & 0 \\
-56 & 28 & -8 & 1 & 0 & \cdots & 0 & 0 \\
\frac{3328}{49553} & -\frac{2645}{594636} & -\frac{256}{743295} & -\frac{1}{594636} & 0 & \cdots & 0 & 0 \\
0 & 0 & 0 & 0 & 0 & \cdots & 0 & 0
\end{array}\right)
\end{aligned}
$$

in $\mathbb{B}$ first four rows come from (33) for $i=5,4,3,2$, fifth row comes from (43), and the last row comes from (29).

The matrix $B_{1}$ is same as defined in Case 1 and the matrices $Z$ and $R$ are defined as

$$
\begin{gathered}
Z=\left(z_{-6}, z_{-5}, \ldots, z_{N+5}, z_{N+6}\right)^{T}, \\
R=\left(0,0,0,0, y^{\prime}(0), y_{r}, D^{T}, y_{l}, 0,0,0,0,0\right)^{T} .
\end{gathered}
$$

The nonsingularity of the coefficients matrix $B$ has been discussed in next section.
3.3. Existence of the Solution. In this section, we discuss the nonsingularity of the coefficients matrix $B$. We observe that the coefficients matrix $B$ is neither symmetric nor diagonally dominant. However it can be shown that $B$ is a nonsingular. Since $B$ is almost a band matrix with half band width 7 , numerical complexity for solving the linear system using Gaussian elimination is about $49(N+9)$ multiplications. For large $N$, the matrix is almost symmetric except the first and last six rows and columns due to the boundary conditions. Therefore we first consider the symmetric part of it, that is, square symmetric matrix $C$ of order $N+3$ defined as

$$
C=\left(\begin{array}{cccccccccccc}
\phi_{1}^{\prime \prime \prime} & \phi_{0}^{\prime \prime \prime} & \phi_{-1}^{\prime \prime \prime} & \phi_{-2}^{\prime \prime \prime} & \phi_{-3}^{\prime \prime \prime} & \phi_{-4}^{\prime \prime \prime} & \phi_{-5}^{\prime \prime \prime} & \phi_{-6}^{\prime \prime \prime} & \cdots & 0 & 0 & 0 \\
\phi_{2}^{\prime \prime \prime} & \phi_{1}^{\prime \prime \prime} & \phi_{0}^{\prime \prime \prime} & \phi_{-1}^{\prime \prime \prime} & \phi_{-2}^{\prime \prime \prime} & \phi_{-3}^{\prime \prime \prime} & \phi_{-4}^{\prime \prime \prime} & \phi_{-5}^{\prime \prime \prime} & \cdots & 0 & 0 & 0 \\
\phi_{3}^{\prime \prime \prime} & \phi_{2}^{\prime \prime \prime} & \phi_{1}^{\prime \prime \prime} & \phi_{0}^{\prime \prime \prime} & \phi_{-1}^{\prime \prime \prime} & \phi_{-2}^{\prime \prime \prime} & \phi_{-3}^{\prime \prime \prime} & \phi_{-4}^{\prime \prime \prime} & \cdots & 0 & 0 & 0 \\
\phi_{4}^{\prime \prime \prime} & \phi_{3}^{\prime \prime \prime} & \phi_{2}^{\prime \prime \prime} & \phi_{1}^{\prime \prime \prime} & \phi_{0}^{\prime \prime \prime} & \phi_{-1}^{\prime \prime \prime} & \phi_{-2}^{\prime \prime \prime} & \phi_{-3}^{\prime \prime \prime} & \cdots & 0 & 0 & 0 \\
0 & 0 & 0 & 0 & 0 & 0 & 0 & 0 & \cdots & \phi_{4}^{\prime \prime \prime} & \phi_{3}^{\prime \prime \prime} & \phi_{2}^{\prime \prime \prime} \\
0 & 0 & 0 & 0 & 0 & 0 & 0 & 0 & \cdots & \phi_{3}^{\prime \prime \prime} & \phi_{2}^{\prime \prime \prime} & \phi_{1}^{\prime \prime \prime}
\end{array}\right)
$$

It can be shown that $C$ is always nonsingular for each value of $N$. However, $B$ is nonsingular for $N \leqslant 1000$. We have checked the nonsingularity of matrix $B$ by different methods. In first method we observe that the determinants of matrix $B$ increase as $N$ increases and it is not zero for $N \leqslant 1000$. So $B$ is nonsingular. The determinants of $B$ at some values of $N$ are shown in Table 1 . In second method we observe that for $N \leqslant 1000$, the eigenvalues of matrix $B$ are nonzero so by [16] matrix $B$ is nonsingular. However for $N>1000$ matrix $B$ may or may not be nonsingular. Therefore we claim that systems (36) and (44) are stable for $N \leqslant 1000$.

\section{Error Estimation}

In this section, we discuss the approximation properties of the numerical interpolating collocation algorithm. Since the scheme (8) reproduces polynomial curve of degree seven so by Dyn [14] scheme has approximation order eight. So the collocation algorithm (14) with septic precision treatments at the endpoints has the power of approximation $O\left(h^{2}\right)$. Here we present our main result for error estimation.

Proposition 8. Suppose the exact solution $y(x) \in C^{8}[0,1]$ and $\left\{z_{i}\right\}$ are obtained by solving either (36) or (44) with 8th order boundary condition at the end points; then

$$
\|\operatorname{err}(x)\|_{\infty}=\left\|Z^{j}-y^{j}\right\|_{\infty}=O\left(h^{2-j}\right), \quad j=0,1,2,
$$

where $j$ denotes the order of derivative.

Proof. Since the order of approximation of subdivision scheme (8) is eight so by using (13), we can write for smooth function $y(x)$ and small $h$ as

$$
\begin{aligned}
y^{\prime \prime \prime}\left(x_{j}\right) & \\
= & \frac{2^{3}}{15039360 h^{3}} \\
& \quad \times\left\{225 y\left(x_{j}-6 h\right)-11520 y\left(x_{j}-5 h\right)\right. \\
& \quad+10952 y\left(x_{j}-4 h\right)+476928 y\left(x_{j}-3 h\right)
\end{aligned}
$$




$$
\begin{aligned}
& -3047987 y\left(x_{j}-2 h\right)+4677632 y\left(x_{j}-h\right) \\
& -4677632 y\left(x_{j}+h\right)+3047987 y\left(x_{j}+2 h\right) \\
& -476928 y\left(x_{j}+3 h\right)-10952 y\left(x_{j}+4 h\right) \\
& \left.+11520 y\left(x_{j}+5 h\right)-225 y\left(x_{j}+6 h\right)\right\} .
\end{aligned}
$$

This can be written as

$$
\begin{aligned}
& y^{\prime \prime \prime}\left(x_{j}\right) \\
&= \frac{2^{3}}{15039360 h^{3}} \\
& \quad \times\left\{225 y_{j-6}-11520 y_{j-5}+10952 y_{j-4}\right. \\
& \quad+476928 y_{j-3}-3047987 y_{j-2}+4677632 y_{j-1} \\
& \quad-4677632 y_{j+1}+3047987 y_{j+2}-476928 y_{j+3} \\
&\left.\quad-10952 y_{j+4}+11520 y_{j+5}-225 y_{j+6}\right\} .
\end{aligned}
$$

Similarly, we have

$$
\begin{aligned}
Z^{\prime \prime \prime}\left(x_{j}\right) & \\
= & \frac{2^{3}}{15039360 h^{3}} \\
\times & \left\{225 z_{j-6}-11520 z_{j-5}+10952 z_{j-4}\right. \\
& +476928 z_{j-3}-3047987 z_{j-2}+4677632 z_{j-1} \\
& \quad 4677632 z_{j+1}+3047987 z_{j+2}-476928 z_{j+3} \\
& \left.-10952 z_{j+4}+11520 z_{j+5}-225 z_{j+6}\right\} .
\end{aligned}
$$

If we define error function $e(x)=Z(x)-y(x)$ and error vectors at the nodes by

$$
e\left(x_{j}\right)=Z\left(x_{j}\right)-y\left(x_{j}+j h\right), \quad-6 \leq j \leq N+6,
$$

or equivalently $e_{j}=Z_{j}-y\left(x_{j}+j h\right),-6 \leq j \leq N+6$, then this implies

$$
\begin{aligned}
& e_{j}^{\prime}=Z_{j}^{\prime}-y^{\prime}(x+j h), \\
& e_{j}^{\prime \prime}=Z_{j}^{\prime \prime}-y^{\prime \prime}(x+j h), \\
& e_{j}^{\prime \prime \prime}=Z_{j}^{\prime \prime \prime}-y^{\prime \prime \prime}(x+j h) .
\end{aligned}
$$

TABLE 1: Determinants of the matrices.

\begin{tabular}{lcc}
\hline$N$ & $C$ & $B$ \\
\hline 10 & $-8667 / 56$ & $1 / 1048870018371741$ \\
50 & -177183 & $1 / 4981270309$ \\
100 & -552709050 & $1 / 1964492$ \\
500 & $-5.033491471916955 \times 10^{36}$ & $4.728852755761116 \times 10^{21}$ \\
1000 & $-4.477989536166907 \times 10^{71}$ & $42069711017699999 \times 10^{40}$ \\
\hline
\end{tabular}

By subtracting (50) from (51), we get

$$
\begin{aligned}
Z^{\prime \prime \prime}\left(x_{j}\right)-y^{\prime \prime \prime}\left(x_{j}\right) \\
=\frac{2^{3}}{15039360 h^{3}} \\
\times\left\{225\left(z_{j-6}-y_{j-6}\right)-11520\left(z_{j-5}-y_{j-5}\right)\right. \\
\quad+10952\left(z_{j-4}-y_{j-4}\right)+476928\left(z_{j-3}-y_{j-3}\right) \\
\quad-3047987\left(z_{j-2}-y_{j-2}\right)+4677632\left(z_{j-1}-y_{j-1}\right) \\
\quad-4677632\left(z_{j+1}-y_{j+1}\right)+3047987\left(z_{j+2}-y_{j+2}\right) \\
\quad-476928\left(z_{j+3}-y_{j+3}\right)-10952\left(z_{j+4}-y_{j+4}\right) \\
\left.\quad+11520\left(z_{j+5}-y_{j+5}\right)-225\left(z_{j+6}-y_{j+6}\right)\right\} .
\end{aligned}
$$

This implies

$$
\begin{aligned}
& e^{\prime \prime \prime}\left(x_{j}\right) \\
& =\frac{2^{3}}{15039360 h^{3}} \\
& \quad \times\left\{225 e_{j-6}-11520 e_{j-5}+10952 e_{j-4}\right. \\
& \quad+476928 e_{j-3}-3047987 e_{j-2}+4677632 e_{j-1} \\
& \quad-4677632 e_{j+1}+3047987 e_{j+2}-476928 e_{j+3} \\
& \left.\quad-10952 e_{j+4}+11520 e_{j+5}-225 e_{j+6}\right\} .
\end{aligned}
$$

By Lemma 5, we get the following expression:

$$
\begin{aligned}
e_{j}^{\prime \prime \prime}=\frac{1}{h^{3}}\{ & \phi_{6}^{\prime \prime \prime} e_{j-6}+\phi_{5}^{\prime \prime \prime} e_{j-5}+\phi_{4}^{\prime \prime \prime} e_{j-4}+\phi_{3}^{\prime \prime \prime} e_{j-3} \\
& +\phi_{2}^{\prime \prime \prime} e_{j-2}+\phi_{1}^{\prime \prime \prime} e_{j-1}+\phi_{0}^{\prime \prime \prime} e_{j}+\phi_{-1}^{\prime \prime \prime} e_{j+1} \\
& +\phi_{-2}^{\prime \prime \prime} e_{j+2}+\phi_{-3}^{\prime \prime \prime} e_{j+3}+\phi_{-4}^{\prime \prime \prime} e_{j+4} \\
& \left.+\phi_{-5}^{\prime \prime \prime} e_{j+5}+\phi_{-6}^{\prime \prime \prime} e_{j+6}\right\}+O\left(h^{8}\right),
\end{aligned}
$$

where $j=0,1,2, \ldots, N$.

By subtracting (1) from (15), we get

$$
Z_{j}^{\prime \prime \prime}-y_{j}^{\prime \prime \prime}=a_{j}\left(Z_{i}-Y_{j}\right) .
$$


This implies

$$
e_{j}^{\prime \prime \prime}=a_{j} e_{j}, \quad 0 \leq i \leq N .
$$

Using (56), we get

$$
\begin{aligned}
& \phi_{6}^{\prime \prime \prime} e_{j-6}+\phi_{5}^{\prime \prime \prime} e_{j-5}+\phi_{4}^{\prime \prime \prime} e_{j-4}+\phi_{3}^{\prime \prime \prime} e_{j-3}+\phi_{2}^{\prime \prime \prime} e_{j-2} \\
& +\phi_{1}^{\prime \prime \prime} e_{j-1}+q_{j} e_{j}+\phi_{-1}^{\prime \prime \prime} e_{j+1}+\phi_{-2}^{\prime \prime \prime} e_{j+2}+\phi_{-3}^{\prime \prime \prime} e_{j+3} \\
& +\phi_{-4}^{\prime \prime \prime} e_{j+4}+\phi_{-5}^{\prime \prime \prime} e_{j+5}+\phi_{-6}^{\prime \prime \prime} e_{j+6}=0,
\end{aligned}
$$

where $q_{j}=\phi_{0}^{\prime \prime \prime}-h^{3} a_{j}$ and $j=0,1,2, \ldots, N$.

As $0 \leq x \leq 1$ and $x_{j}=j h, j=0,1,2, \ldots, N$ so $e_{0}, e_{1}, \ldots, e_{N}$ are nonzero while $e_{-6}, e_{-5}, \ldots, e_{-1}$ and $e_{N+1}, e_{N+2}, \ldots, e_{N+6}$ are zero because they lie outside the interval $[0,1]$. Let us define these (the left and right end) error values as

$$
e_{j}= \begin{cases}\max _{0 \leq k \leq 7}\left\{\left|e_{k}\right|\right\} O\left(h^{8}\right), & -6 \leq j \leq 0, \\ \max _{N-7 \leq k \leq N}\left\{\left|e_{k}\right|\right\} O\left(h^{8}\right), & N \leq j \leq N+6 .\end{cases}
$$

Thus system (59) is equivalent to

$$
\left(B+O\left(h^{6}\right)\right) E=0,
$$

where $B+O\left(h^{6}\right)$ is the matrix obtained by deleting the first and last six rows and columns of the matrix $B$, where

$$
E=\left(e_{-6}, e_{-5}, e_{-4}, \ldots, e_{N+4}, e_{N+5}, e_{N+6}\right)^{T} .
$$

By using (7)

$$
\begin{aligned}
\left(B+O\left(h^{6}\right)\right) E & =O\left(h^{8}\right)\left\|Z\left(x_{j}\right)-y\left(x_{j}\right)\right\| \\
& =O\left(h^{8}\right)\|E\|=O\left(h^{8}\right) .
\end{aligned}
$$

Hence, for small $h$, the coefficients matrix $B+O\left(h^{6}\right)$ will be invertible and thus using the standard result from linear algebra, we have

$$
\|E\| \leq\left(\frac{\left\|B^{-1}\right\|}{1-O\left(h^{6}\right)} O\left(h^{8}\right)\right)=O\left(h^{2}\right) .
$$

This completes the proof.

The above discussion suggests that the approximations of the solution computed by the method developed in pervious section are second order accurate approximations. This suggestion is supported by the numerical experiments given in the next section

\section{Numerical Examples and Discussions}

In this section, the numerical collocation algorithm based on 8-point interpolating subdivision scheme described in Section 3, with the 8th order boundary conditions at the end points, is tested on the two-point third order boundary value problems. Absolute errors in the analytical solutions are also calculated. For the sake of comparisons, we also tabulated the results in this section.
Example 1. Consider the boundary value problem

$$
y^{\prime \prime \prime}(x)=y(x)-3 e^{x}, \quad 0<x<1,
$$

with boundary conditions $y^{\prime}(0)=0, y(1)=0, y(0)=1$. The analytical solution of this problem is

$$
y(x)=(1-x) e^{x}
$$

By using the collocation algorithm for $N=10$, we get following solution of the above problem: $Z_{j}=\sum_{i=-6}^{16} z_{i} \phi(j-i)$, where the values of $\left\{z_{-6}, z_{-5}, \ldots, z_{5}, z_{16}\right\}$ by using (36) are

$$
\{24.5275284967525,0.690493373832105 \text {, }
$$$$
0.811531107670859 \text {, }
$$$$
0.901997043612006,0.962835576958380
$$$$
0.995101658947808,1,0.978925359857389 \text {, }
$$$$
0.933503956247400,0.865636023693632 \text {, }
$$$$
\text { 0.797539554103408, 0.771795250759200, }
$$$$
\text { 0.651392727341382, 0.519777983609805, }
$$$$
\text { 0.380902186129123, 0.199271785305208, 0, }
$$$$
-0.131140299007903,-0.247662275449558 \text {, }
$$$$
-0.342309352611581,-0.406997945734322 \text {, }
$$$$
-0.432827137019005,-0.413625213339484\}
$$

and by using (44) are

$$
\{0.8188703235427,0.8701325409827,0.9139995186898 \text {, }
$$

$$
\text { 0.9498836119009, 0.9768946175522, 0.9940000846665, }
$$

$$
\text { 1, 0.9934991816499, 0.9728768806892, 0.9362530908960, }
$$$$
0.8814510666459,0.8059555490326,0.7068662002854 \text {, }
$$$$
0.5808456281783,0.4240613657868,0.2321210447258,0 \text {, }
$$$$
-0.2780394553965,-0.6085378480019 \text {, }
$$$$
-0.9989382153934,-1.457696393548 \text {, }
$$$$
1.993136716176,-2.54764506053343\} \text {. }
$$

By using two different boundary treatments presented in Section 3, we obtained two different solutions which are presented in Table 2 along with their absolute errors. The graphical representations of the analytic and approximate solutions of the above problem are shown in Figure 2. Figure 2(a) represents the comparison of analytic and approximate solutions obtained by (36) while analytic and approximate solutions obtained by (44) are shown in Figure 2(b). From this table and figure, we observe that the solution obtained by (44) is significantly better than the solution 
TABLE 2: Solutions and error estimation of Example 1.

\begin{tabular}{|c|c|c|c|c|c|}
\hline$x_{j}$ & Analytic solution $Y$ & $\begin{array}{c}\text { Approximate solution } \\
Z \text { by (36) }\end{array}$ & $\begin{array}{c}\text { Approximate solution } \\
Z \text { by (44) }\end{array}$ & $\begin{array}{l}\text { Absolute error } \\
\text { by (36) }\end{array}$ & $\begin{array}{c}\text { Absolute error } \\
\text { by }(44)\end{array}$ \\
\hline 0.0 & 1 & 1 & 0.0000 & 0 & 0 \\
\hline 0.1 & 0.9946538262 & 0.978925359857389 & 0.9934991878 & 0.0157284663 & 0.00115463837 \\
\hline 0.2 & 0.9771222064 & 0.933503956247400 & 0.9728768936 & 0.0436182502 & 0.00424531280 \\
\hline 0.3 & 0.9449011656 & 0.865636023693632 & 0.9362531105 & 0.0792651419 & 0.00864805513 \\
\hline 0.4 & 0.8950948188 & 0.797539554103408 & 0.8814510903 & 0.0975552647 & 0.01364372850 \\
\hline 0.5 & 0.8243606355 & 0.771795250759200 & 0.8059555784 & 0.0525653847 & 0.0184050571 \\
\hline 0.6 & 0.7288475200 & 0.651392727341382 & 0.7068662336 & 0.0774547927 & 0.0219812864 \\
\hline 0.7 & 0.6041258121 & 0.519777983609805 & 0.5808456679 & 0.0843478285 & 0.0232801441 \\
\hline 0.8 & 0.4451081856 & 0.380902186129123 & 0.42406141196 & 0.06420599959 & 0.0210467736 \\
\hline 0.9 & 0.2459603111 & 0.199271785305208 & 0.23212109670 & 0.0466885258 & 0.0138392144 \\
\hline 1.0 & 0 & 0 & 0.0000 & 0.000 & 0.000 \\
\hline
\end{tabular}

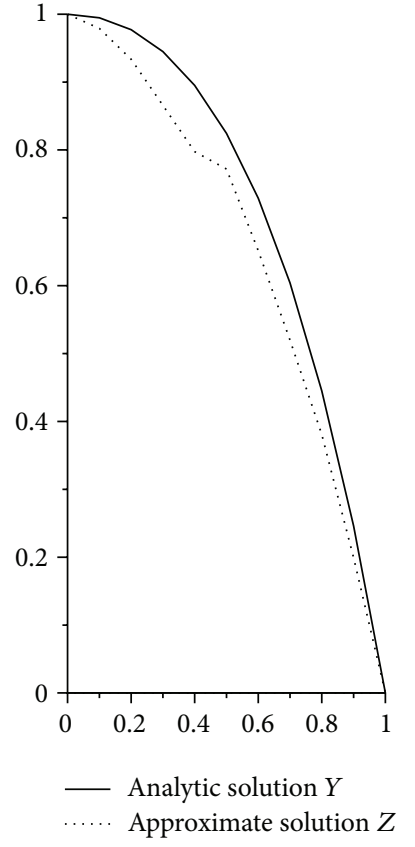

(a)

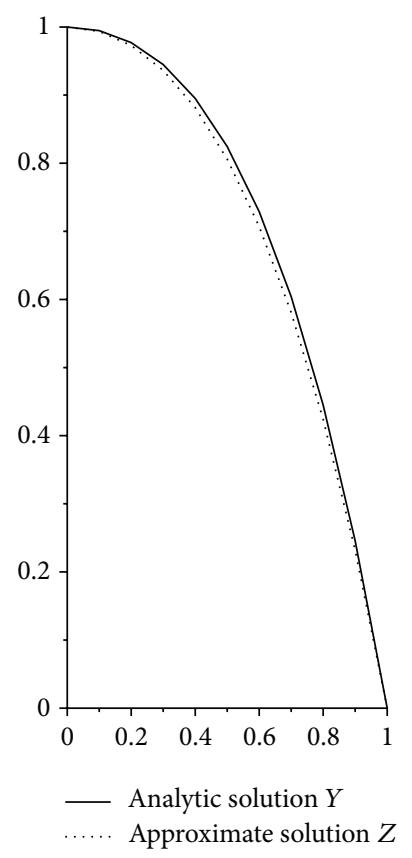

(b)

FIGURE 2: Comparison between analytic and approximating solutions.

obtained by (36). So our claim; that is, the approximate solution can be improved by adjusting boundary treatment, is justified. The maximum absolute errors in the solutions obtained by (36) and (44) at step size 10 are $9.755 \times 10^{-2}$ and $2.328 \times 10^{-2}$, respectively.

Example 2. Consider the following third order boundary values problem:

$$
y^{\prime \prime \prime}(x)=x y(x)+\left(x^{3}-2 x^{2}-5 x-3\right) e^{x}, \quad 0<x<1,
$$

with boundary conditions $y(0)=0=y(1), y^{\prime}(0)=1$. Its exact solution is

$$
y(x)=x(1-x) e^{x}
$$

By the homogeneous process of the boundary condition, let $u(x)=y(x)-x(1-x)$; then above problem can be transformed into its equivalent form

$$
\begin{array}{r}
u^{\prime \prime \prime}(x)=x u(x)+x^{2}(1-x)+\left(x^{3}-2 x^{2}-5 x-3\right) e^{x}, \\
0<x<1, \quad u(0)=u(1)=u^{\prime}(0)=0 .
\end{array}
$$

The solutions of this problem and their absolute errors obtained by two different boundary treatments are shown in Table 3. The graphical representations of the analytic and approximate solutions are shown in Figure 3. Figure 3(a) represents the comparison of analytic and approximate 


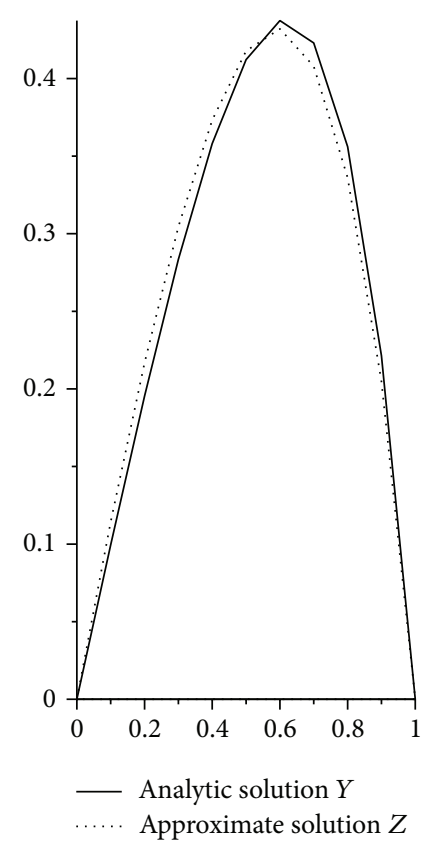

(a)

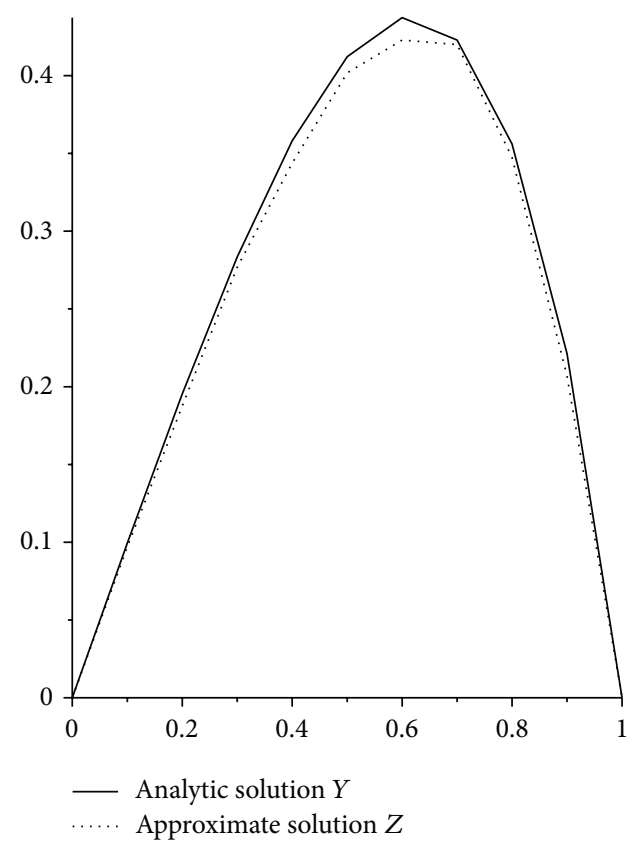

(b)

FIGURE 3: Comparison between analytic and approximating solutions.

TABLE 3: Solutions and error estimation of Example 2.

\begin{tabular}{cccccc}
\hline$x_{j}$ & Analytic solution $Y$ & $\begin{array}{c}\text { Approximate solution } \\
Z \text { by (36) }\end{array}$ & $\begin{array}{c}\text { Approximate solution } \\
Z \text { by (44) }\end{array}$ & $\begin{array}{c}\text { Absolute error } \\
\text { by (36) }\end{array}$ & $\begin{array}{c}\text { Absolute error } \\
\text { by (44) }\end{array}$ \\
\hline 0.0 & 0 & 0 & 0.0000 & 0.000 & 0.000 \\
0.1 & 0.09946538262 & 0.1142745006 & 0.09710744670 & 0.01480911798 & 0.00235793592 \\
0.2 & 0.1954244413 & 0.2168561360 & 0.1876024920 & 0.0214316947 & 0.0078219493 \\
0.3 & 0.2834703497 & 0.3045203260 & 0.2767792681 & 0.0210499763 & 0.0066910816 \\
0.4 & 0.3580379275 & 0.3732002884 & 0.3437107723 & 0.0151623609 & 0.0143271552 \\
0.5 & 0.4121803178 & 0.4177996505 & 0.4018316295 & 0.0056193327 & 0.0103486883 \\
0.6 & 0.4373085120 & 0.4319808639 & 0.4229368864 & 0.0053276481 & 0.0143716256 \\
0.7 & 0.4228880685 & 0.4079258312 & 0.4199575084 & 0.0149622373 & 0.0029305601 \\
0.8 & 0.3560865485 & 0.3360647276 & 0.3473442367 & 0.0200218209 & 0.0087423118 \\
0.9 & 0.2213642800 & 0.2047684968 & 0.2069998108 & 0.0165957832 & 0.0143644692 \\
1.0 & 0 & 0 & 0.0000 & 0.000 & 0.000 \\
\hline
\end{tabular}

solutions obtained by (36). Figure 3(b) represents the comparison of analytic and approximate solutions obtained by (44). We observe that the solution obtained by (44) has less absolute error than that of the solution obtained by (36). Again this supports our claim.

Comparison. The maximum absolute errors in the solutions obtained by (36) and (44) at step size 10 are $2.143 \times 10^{-2}$ and $1.437 \times 10^{-2}$, respectively. Caglar et al. [17] obtained the same maximum absolute errors but at the step size 32 and 50, respectively. Therefore we conclude that our method is more efficient than that of Caglar et al.

\section{Conclusion and Future Work}

In this work, we present an interpolatory symmetric subdivision algorithm for the numerical solution of third order linear problems. Septic polynomials were used for the adjustment of boundary conditions at the end points. We established collocation method and obtained stable system of linear equations which can be solved by any well-known numerical method. The numerical result showed that the adjustment of boundary conditions at the end points influence the accuracy of approximate solution. That is, the accuracy of the solution can be improved by the proper adjustment of boundary conditions. So our algorithm has flexibility 
to improve the results by adjusting boundary conditions. The automatic selection and adjustment of the boundary conditions which improve the approximation order of the solution is possible future research direction.

\section{Conflict of Interests}

The authors declare that there is no conflict of interests regarding the publication of this paper.

\section{Acknowledgments}

The authors thank the anonymous reviewer whose valuable comments greatly improved this work. This work is supported by Indigenous PhD Scholarship Scheme of Higher Education Commission (HEC), Pakistan.

\section{References}

[1] G. de Rham, "Sur une courbe plane," Journal de Mathématiques Pures et Appliquées, vol. 35, pp. 25-42, 1956.

[2] N. Dyn, D. Levin, and J. A. Gregory, "A 4-point interpolatory subdivision scheme for curve design," Computer Aided Geometric Design, vol. 4, no. 4, pp. 257-268, 1987.

[3] G. Deslauriers and S. Dubuc, "Symmetric iterative interpolation processes," Constructive Approximation, vol. 5, no. 1, pp. 49-68, 1989.

[4] G. Mustafa and N. A. Rehman, "The mask of $(2 b+4)$-point $n$-ary subdivision scheme," Computing: Archives for Scientific Computing, vol. 90, no. 1-2, pp. 1-14, 2010.

[5] M. Aslam, G. Mustafa, and A. Ghaffar, “( $2 n-1)$-point ternary approximating and interpolating subdivision schemes," Journal of Applied Mathematics, vol. 2011, Article ID 832630, 12 pages, 2011.

[6] G. Mustafa, J. Deng, P. Ashraf, and N. A. Rehman, "The mask of odd points $n$-ary interpolating subdivision scheme," Journal of Applied Mathematics, vol. 2012, Article ID 205863, 20 pages, 2012.

[7] G. Mustafa and F. Khan, "A new 4-point $C^{3}$ quaternary approximating subdivision scheme," Abstract and Applied Analysis, vol. 2009, Article ID 301967, 14 pages, 2009.

[8] G. Mustafa, P. Ashraf, and J. Deng, "Generalized and unified families of inter-polating schemes," Numerical MathematicsTheory Methods and Applications, vol. 7, no. 2014, pp. 193-213, 2014.

[9] G. Mustafa and I. P. Ivrissimitzis, "Model selection for the Dubuc-Deslauriers family of subdivision schemes," in Proceedings of the 14th IMA Conference on Mathematics of Surfaces, University of Birmingham, Birmingham, UK, September 2013.

[10] R. Qu and R. P. Agarwal, "Solving two point boundary value problems by interpolatory subdivision algorithms," International Journal of Computer Mathematics, vol. 60, no. 3-4, pp. 279-294, 1996.

[11] R. Qu and R. P. Agarwal, "A subdivision approach to the construction of approximate solutions of boundary-value problems with deviating arguments," Journal of Computers \& Mathematics with Applications, vol. 35, no. 11, pp. 121-135, 1998.

[12] R. Qu, "Curve and surface interpolation by subdivision algorithms," Computer Aided Drafting Design and Manufacturing, vol. 4, no. 2, pp. 28-39, 1994.
[13] R. B. Qu and R. P. Agarwal, "A cross difference approach to the analysis of subdivision algorithms," Neural, Parallel \& Scientific Computations, vol. 3, no. 3, pp. 393-416, 1995.

[14] N. Dyn, "Tutorial on multiresolution in geometric modelling summer school lecture notes series," in Mathematics \& Visualization, I. Armin, A. Ewald, and S. F. Michael, Eds., Springer, 2002.

[15] C. Conti and K. Hormann, "Polynomial reproduction for univariate subdivision schemes of any arity," Journal of Approximation Theory, vol. 163, no. 4, pp. 413-437, 2011.

[16] G. Strang, Linear Algebra and Its Applications, Cengage Learning India Private Limited, 4th edition, 2011.

[17] H. N. Caglar, S. H. Caglar, and E. H. Twizell, "The numerical solution of third-order boundary-value problems with fourthdegree B-spline functions," International Journal of Computer Mathematics, vol. 71, no. 3, pp. 373-381, 2007. 


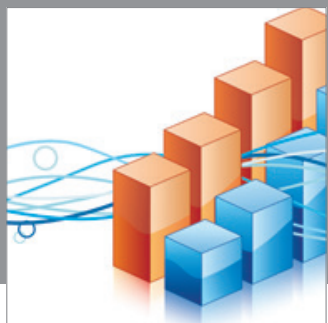

Advances in

Operations Research

mansans

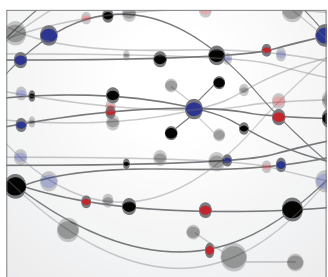

The Scientific World Journal
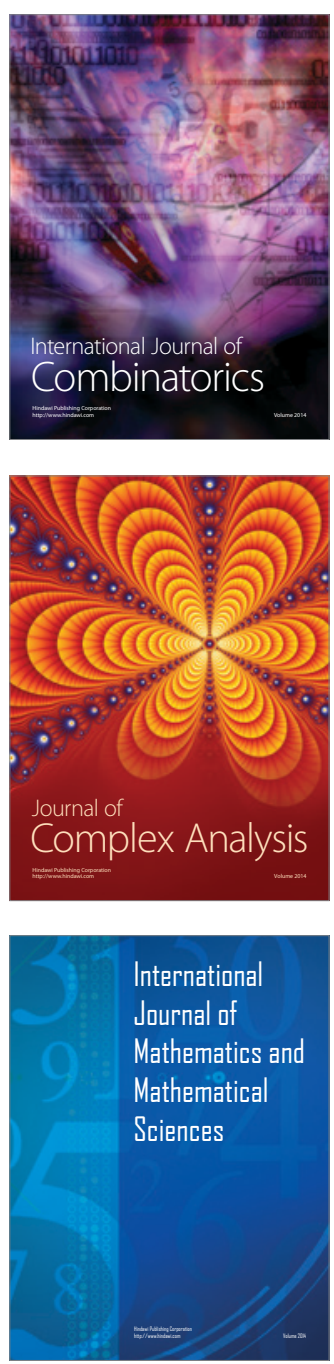
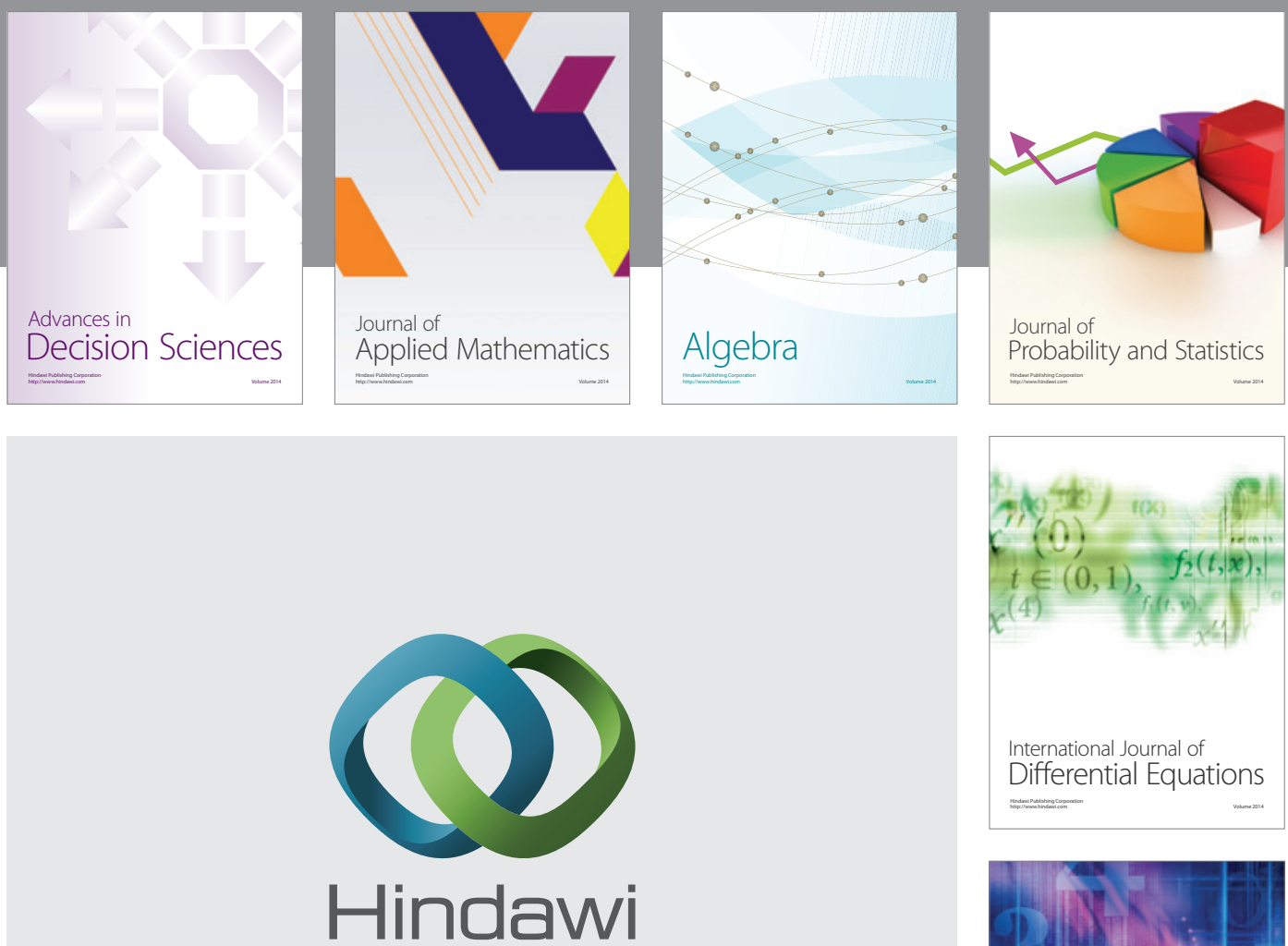

Submit your manuscripts at http://www.hindawi.com
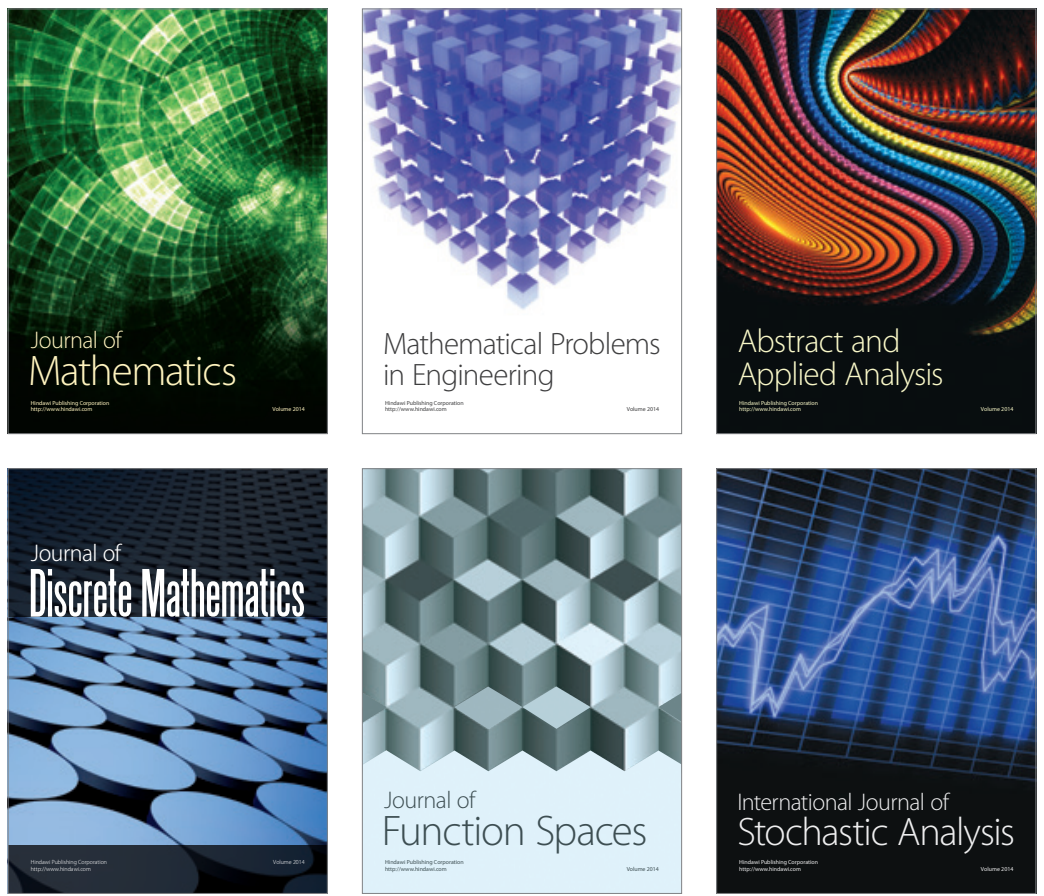

Journal of

Function Spaces

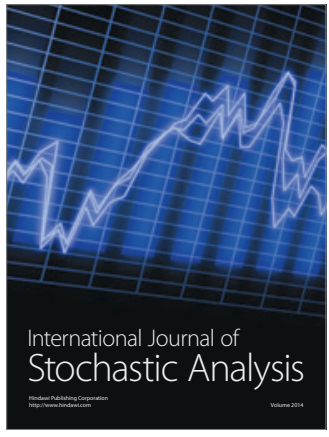

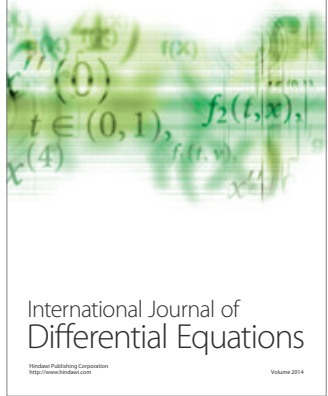
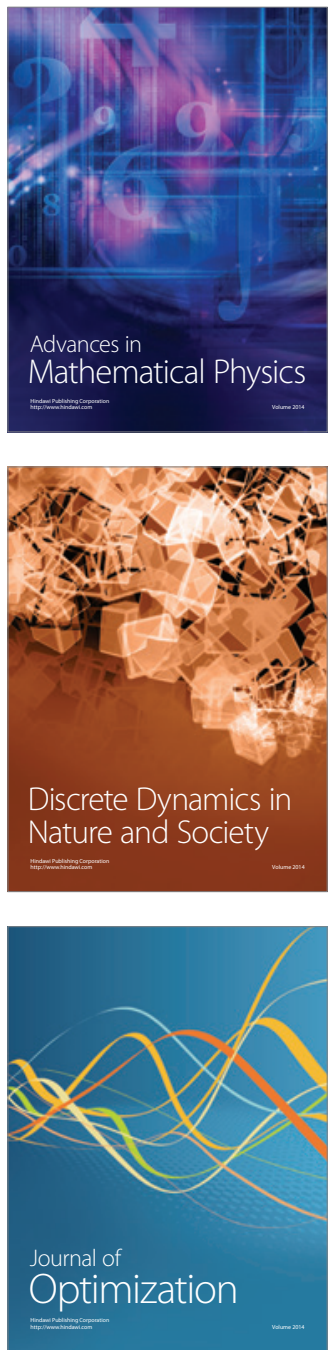